\title{
SOLUTION OF THE WORD PROBLEM FOR CERTAIN TYPES OF GROUPS I
}

\author{
by J. L. BRITTON \\ (Received 12th December, 1955)
}

Introduction. The main result of this series of papers is a theorem on the free product of groups (Theorem 1) which formed part of a doctoral thesis. $f$ This theorem has an immediate application to the word problem (Theorem 2). Usually the word problem refers to a finite system of generators and a finite number of defining relations, but in this context it is more natural to allow an infinite number of generators and defining relations. This (infinite) word problem is not solvable in general (Example 2).

The results obtained consist roughly of an extension of the results of $\mathrm{V}$. A. Tartakovskii ([7], [8], [9]) on the case of a free product of a finite number of cyclic groups with a finite number of additional defining relations, to the case of a free product of a possibly infinite number of arbitrary groups with a possibly infinite number of additional defining relations.

The method is entirely different. For the finite cyclic case it is found, in the notation of Tartakovskii [8], that the condition $\delta<1 / 6$ is sufficient for the word problem to be solvable. The method does not however give a counterpart to the condition $k>6$.

In the case when all the constituent groups of the free product are cyclic, a result on the infinite word problem has been obtained by Stender [6]. This result is not actually correct as it stands (Example 3), but it can easily be made so by adding a further assumption (namely, (3) of Lemma 2.32), for it is then a consequence of Theorem 2.

Condition (2B) cannot be omitted from Theorem 2 because a system exists which satisfies every condition of Theorem 2 except this one but for which the word problem is not solvable (Lemma 2.33).

\section{[Added 21st March 1956]}

Condition (2A) cannot of course be omitted, but it is shown in the Appendix that (2C) can in fact be omitted without destroying the validity of Theorem 2.

The present paper is concerned only with the application of Theorem 1 to the word problem. The proof of Theorem 1 itself is postponed.

\section{Preliminary definitions. Statement of the Main Theorem.}

1.1. Let $\Pi$ be the free product of the set of groups $\left\{G_{\gamma} \mid \gamma \in \Gamma\right\}$ where the index set $\Gamma$ may be infinite. No restriction is placed on the groups $G_{\gamma}$, which will be called the constituent groups of $\Pi$. We denote elements of $\Pi$ by capital letters and write the group multiplicatively so that the product of two elements $X, Y$ is $X . Y$. Let $I$ be the unit element. Every element $U$ of $\Pi$ except $I$ has a unique representation, called the normal form of $U$, of the type

$$
U=X_{y(1)} \cdot X_{Y(2)} \ldots . X_{\gamma(\tau)}
$$

where $r \geqslant 1, I \neq X_{\gamma^{(i)}} \in G_{\gamma^{(i)}}(i=1,2, \ldots, r)$ and $\gamma(i) \neq \gamma(i+1)(i=1,2, \ldots, r-1)$. We denote $r$ by $l(U)$ and call it the length of $U$. The length $l(I)$ of $I$ is defined to be zero. Elements of unit

$\dagger \mathrm{Ph} . \mathrm{D}$. thesis, with the same title as the present paper, presented to Manchester University (1953). I am very grateful to Dr. B. H. Neumann who supervised the work for this thesis. 
length are called components and will be denoted by small letters. If $x, y$ are elements of the same constituent group we write $x \sim y$. If not we write $x \sim \sim^{\prime} y$.

The Dot Convention

If $X$ is a product $X_{1}, X_{2} \ldots \ldots X_{m}$ of elements of $\Pi$ and if $l(X)=\sum_{i=1}^{m} l\left(X_{i}\right)$ we shall write $X=X_{1} X_{2} \ldots X_{m}$; that is, we omit the dots. In particular the dots can be omitted from a normal form. The expression (1.11) now becomes

We shall also write

$$
U=x_{\gamma(1)} x_{Y(2)} \ldots x_{Y(r)} \text {. }
$$

$$
x_{\gamma(1)}=\operatorname{In}(U), \quad x_{\gamma(r)}=\operatorname{Fin}(U) .
$$

Now let $X, Y$ be elements of $\Pi$ different from $I$. We define the number $\beta(X, Y)$ of cancellations in the product $X, Y$ and the number of amalgamations, written $\varepsilon(X, Y)$, as follows:

Let

$$
X=a_{1} a_{2} \ldots a_{m}, \quad Y=b_{1} b_{2} \ldots b_{n} .
$$

Then

$$
\beta(X, Y)= \begin{cases}0 & \text { if } a_{m} \cdot b_{1} \neq I, \\ \beta & \text { if } a_{m} \cdot b_{1}=I,\end{cases}
$$

where $\beta$ is the largest integer for which $a_{m-\beta+1} \ldots a_{m} . b_{1} \ldots b_{\beta}=I$, and

Put

$$
\varepsilon(X, Y)= \begin{cases}1 & \text { if } \beta<m, \beta<n \text { and } a_{m-\beta} \sim b_{\beta+1}(\beta=\beta(X, Y)), \\ 0 & \text { otherwise. }\end{cases}
$$

Note that

$$
\alpha(X, Y)=\beta(X, Y)+\varepsilon(X, Y) .
$$

$$
l(X . Y)=l(X)+l(Y)-2 \beta(X, Y)-\varepsilon(X, Y) .
$$

1.2. In the following theorem, $\Omega$ denotes a subset of $\Pi$ in which every element $W$ has length at least 2 and $\operatorname{In}(W) \sim^{\prime} \operatorname{Fin}(W)$. If $W=a_{1} a_{2} \ldots a_{n}$, the $n$ elements

$$
a_{r} a_{r+1} \ldots a_{n} a_{1} a_{2} \ldots a_{r-1} \quad(r=1,2, \ldots, n)
$$

are called the cyclic arrangements of $W$. Let $\Omega^{*}$ consist of the cyclic arrangements of all elements in $\Omega$ and their inverses. If $U \varepsilon \Omega *$, write

$$
\alpha(U)=\operatorname{Max} \alpha\left(\bar{U}^{\kappa}, V\right)
$$

where $\bar{U}$ is a cyclic arrangement of $U, \kappa= \pm 1, V \epsilon \Omega^{*}$ and $\bar{U}^{\kappa} . V \neq I$, and all possible choices of $\bar{U}, \kappa$ and $V$ are taken.

We can now formulate the Main Theorem.

THEOREM 1. Let $\Omega$ be a possibly infinite subset of a free product $\Pi$ in which every element $W$ has length at least 2 and $\operatorname{In}(W) \sim \operatorname{Fin}(W)$. Let $\Omega^{*}$ be the set of the cyclic arrangements of the elements of $\Omega$ and their inverses. If, for every choice of elements $U, V$ in $\Omega^{*}$, either $U . V=I$ or

$$
6 \alpha(U, V)<\operatorname{Min}(l(U), l(V)),
$$

then the elements of the normal subgroup [S] of II generated by $\Omega$ have the following properties:

A. Every element $Y$ except $I$ has length at least $l_{0}=\operatorname{Min}_{W \in \Omega} l(W)$, and $l(Y)=l_{0}$ implies $Y_{\epsilon} \Omega^{*}$. In particular, the intersection of [S] with any constituent group consists only of the unit element $I$. 
B. The normal form of $Y$ has a factorization $Y=X K Z$, where there exists an element $V$ of $\Omega^{*}$ with normal form $V=K^{\prime} K$ and

$$
l(K) \geqslant l(\mathrm{~V})-3 \alpha(V)-1
$$

Moreover, equality in $(\mathbf{1 \cdot 2 3 )}$ implies

$$
\operatorname{In}\left(K^{\prime}\right) \sim \operatorname{In}(Z)
$$

and

$$
\operatorname{Fin}\left(K^{\prime}\right) \sim \operatorname{Fin}(X) .
$$

C. The element $X . K^{\prime-1} . Z$ in $[\Omega]$ has length strictly greater than $l(Y)$. Thus

$$
\begin{array}{ll}
\text { either } & l(K)>\frac{1}{2} l(V), \\
\text { or } & l(K)=\frac{1}{2} l(V) \text { and }(1 \cdot 24) \text { or }(1 \cdot 25) \text { holds, } \\
\text { or } & l(K)=\frac{1}{2}(l(V)-1) \text { and both }(1 \cdot 24) \text { and }(1 \cdot 25) \text { hold. }
\end{array}
$$

An Alternative Condition (1.26)

The theorem remains true if we replace $(1 \cdot 22)$ by the weaker condition

$$
1+4 \alpha(U, V)<\operatorname{Min}(l(U), l(V))
$$

but at the same time restrict cancellations between certain sets of three elements of $\Omega^{*}$.

The precise form of this restriction will not be given here because it is rather lengthy to state and it will not be required in the present paper.

\section{Application to the word problem.}

2.1. Suppose that a set $S$ of generators and a set $R$ of defining relations are explicitly given, where $S$ and $R$ may be infinite sets, and it is asked whether or not the word problem is solvable for the system $\{S, R\}$. We shall see how the Main Theorem allows one to give a positive answer when the system satisfies certain conditions, but let us first clarify the problem a little.

An infinite set is regarded as explicitly given if a finite process $\dagger$ is provided for determining whether or not any given "mathematical object" is an element of the set. In the system $\{S, R\}$ the set $S$ consists of certain identifiable symbols and the elements of $R$ are relations of the form $w=1$, where $w$ is a word in these symbols, that is, a finite sequence of symbols each with an index +1 or -1 . A word $w$ is called a defining word if it is the left hand side of a defining relation. Let $\bar{R}$ be the set of defining words and let $W$ be the set of all words. We include in $W$ the "empty word" which contains no symbols. Two words $w_{1}, w_{2}$ are called equivalent if one can be obtained from the other by a finite number of deletions or insertions of words of the form $x^{+1} x^{-1}$ or $x^{-1} x^{+1}(x \in S)$. The multiplication and inverse operations for words are defined in the usual way and if $w$ is any word equivalent to a word of the form $\prod_{i=1}^{m} t_{i}^{-1} w_{k(i)}^{\epsilon(i)} t_{i}$, where $t_{i} \epsilon W, w_{k(i)} \epsilon \bar{R}$ and $\epsilon(i)= \pm 1$, we say that the relation $w=1$ follows from the defining relations. We say that the word problem is solvable for the system $\{S, R\}$ if there is a finite process for deciding whether or not the relation $w=1$ follows from the defining relations, where $w$ is any given word.

It is known that there is a unique abstract group $G(S, R)$ corresponding to any given set $S$ of generators with a set $R$ of defining relations having the following properties:

(i) There is a mapping $\theta$ of $S$ into $G(S, R)$ and $G(S, R)$ is generated by all the elements $s \theta(s \in S)$.

$\dagger$ Defined in Post [5] and Turing [11]. An equivalent definition is given in Church [1]. 
(ii) If $w$ is a defining word, then $w \theta$ is the unit element of $G(S, R)$, where $w \theta$ is defined in an obvious manner.

(iii) Any group $H$ satisfying (i) and (ii) is a homomorphic image of $G(S, R)$ and if $\theta^{\prime}$ is the mapping associated with $H$, then $s \theta$ is mapped by the homomorphism onto $s \theta^{\prime}$, for all $s$ in $S$.

(iv) $w=1$ follows from the defining relations if and only if $w \theta$ is the unit element of $G(S, R)$.

2.2. It will be shown that the word problem is solvable for an explicitly given system $\{S, R\}$ satisfying the following conditions (1) to (5). This result will be proved, and the conditions (1) to (5) summarized, in Theorem 2.

(1) $S$ can be decomposed into a union of disjoint subsets $S_{\gamma}(\gamma \in \Gamma)$ and $R$ into a union of disjoint subsets $R^{\prime}$ and $R_{\gamma}(\gamma \epsilon \Gamma)$, where $R_{\gamma}$ consists of those elements of $R$, if any, which involve only the elements of $S_{\gamma}$. The sets $R^{\prime}, R_{\gamma}$ and $S_{\gamma}$ are required to be given explicitly, and, given $s \in S$, it is required that the set $S_{y}$ which contains $s$ can be obtained in a finite number of steps.

(2) The word problem is solvable for each system $\left\{S_{\gamma}, R_{\gamma}\right\}$.

In the remaining conditions, let $G_{\gamma}=G\left(S_{\gamma}, R_{\gamma}\right)$ and let $\Pi$ be the free product of the groups $G_{y}(\gamma \in \Gamma)$. We shall say that a word in $S$ (i.e., a word formed from the symbols in the set $S$ ) is $\Pi$-reduced if it is a word of the form

$$
w_{\gamma(1)} w_{\gamma(2)} \ldots w_{\gamma(n)}
$$

where $n \geqslant l$ and $w_{y(i)}$ is a word in $S_{\gamma^{(i)}}$ such that the relation $w_{y(i)}$ does not follow from the defining relations in the set $R_{\gamma(i)}$ and also $\gamma(i) \neq \gamma(i+1)(i=1,2, \ldots, n-1)$.

Every word $w$ in $S$ represents an element $W$ of $\Pi$. If we define the $\Pi$-length of the word $w$ to be $l(W)$, it is clear that the word $(2 \cdot 21)$ has $\Pi$-length $n$. It is easy to describe a process $\mathcal{P}_{1}$ which when applied to an arbitrary word $w$ in $S$ yields a word $w^{\prime}$ which represents the same element of $\Pi$ and which is either $\Pi$-reduced or equivalent to the "empty word". We first bracket together the symbols in the word $w$ so that all symbols in a bracket belong to the same set $S_{\gamma}$ and symbols in adjacent brackets belong to different sets. Let the symbols in the $i$ th bracket belong to $S_{\gamma(i)}$ and form the word $w_{i}$, so that $w=w_{1} w_{2} \ldots w_{k}$ if there are $k$ pairs of brackets altogether. We next erase each $w_{i}$ for which the relation $w_{i}=1$ follows from the defining relations in the set $R_{\gamma(i)}$. We can always do this because the word problem is solvable for each system $\left\{S_{\gamma}, R_{\gamma}\right\}$. After a finite number of repetitions of these steps we arrive at the required word $w^{\prime}$.

Two words $w_{1}, w_{2}$ in $S$ will be called conjugate if $w_{1}$ is equivalent to $t^{-1} w_{2} t$ for some word $t$ in $S$, and a $\Pi$-reduced word $(2 \cdot 21)$ will be called a short word if either $n=1$, or $n \neq 1$ and $\gamma(1) \neq \gamma(n)$. It is easy to describe a finite process $P_{2}$ which when applied to a $\Pi$-reduced word $w^{\prime}$ yields a short word $w^{\prime \prime}$ such that $w^{\prime}$ and $w^{\prime \prime}$ are conjugate.

We can now formulate the remaining conditions.

(3) If a defining relation $w=1$ is in $R^{\prime}$, then the word $w^{\prime}=\mathrm{P}_{1}(w)$ is not equivalent to the empty word, and the word $w^{\prime \prime}=\mathcal{P}_{2}\left(w^{\prime}\right)$ has $\Pi$-length at least 2 . Further, if $\Omega$ denotes the subset of $\Pi$ consisting of all elements represented by such words $w^{\prime \prime}$, then condition (1.22) (or (1.26)) of the Main Theorem is satisfied.

We remark that the number of cancellations and amalgamations between two given elements of $\Omega^{*}$ can always be calculated because of condition (2).

It is well known that $\Pi \cong G(S, A)$, where $A=\cup R_{\gamma}(\gamma \in \Gamma)$, and that $\Pi /[\Omega] \cong G(S, R)$. 
(4) There is a finite process for deciding whether or not a given word in $S$ represents some element of the set $\Omega_{1}^{*}$, defined as follows :

Definition $(2 \cdot 22)$. In the notation of the Main Theorem, if $U_{\epsilon} \Omega^{*}$, denote by $U_{1}$ the product of the last $r(U)$ components of the normal form of $U$, where

$$
r(U)=\operatorname{Max}\left(l(U)-3 \alpha(U)-1,\left[\frac{1}{2} l(U)\right]\right) .
$$

Then $\Omega_{1}^{*}$, is the set of all elements $U_{1}$, for $U_{\epsilon} \Omega^{*}$.

We remark that $U_{1}$ uniquely determines $U$, for if $U, U^{\prime} \Omega^{*}$ and $U=V U_{1}, U^{\prime}=V^{\prime} U_{1}$, then $\alpha\left(U, U^{\prime}-1\right) \geqslant l\left(U_{1}\right) \geqslant\left[\frac{1}{2} l(U)\right]>\alpha(U)$. By the definition of $\alpha(U)$, this implies $U \cdot U^{\prime-1}=I$, so that $U=U^{\prime}$.

(5) If a given word in $S$ does represent an element of $\Omega_{1}^{*}$, then there is a finite process for determining some word which represents the corresponding element of $\Omega^{*}$.

Theorem 2. Let the conditions of the Main Theorem be satisfied, either with (1.22) or with $(1 \cdot 26)$. Then the word problem is solvable for the group $\Pi /[\Omega]$ provided that

(2A) the word problem is solvable for each constituent group $G_{\gamma}$,

(2B) the elements of $\Omega_{1}^{*}$ are recognisable, where $\Omega_{1}^{*}$ has the meaning of Definition $(2 \cdot 22)$,

(2C) given any element of $\Omega_{1}^{*}$, the corresponding element of $\Omega^{*}$ can be determined in a finite number of steps.

Proof. Given any word $w$ in the generators of $\Pi /[\Omega]$, we have to give a finite process to determine whether or not the corresponding element of $\Pi /[\Omega]$ is the unit element. Equivalently, we may give a finite process to determine whether or not the corresponding element $X$ of $\Pi$ lies in [ $\Omega]$.

We first determine a word $w^{\prime}$ which represents $X$ and is either a $\Pi$-reduced word of the form $(2 \cdot 21)$, with $\Pi$.length $n$, or is equivalent to the empty word. In the second case $X_{\epsilon}[\Omega]$, so we may assume that the first case is applicable.

Next we examine each "subword" of $w^{\prime}$ of the form $v_{i j}=w_{\gamma(i)} w_{\gamma(i+1)} \ldots w_{\gamma(j)}$ and determine whether or not it represents an element of $\Omega_{1}^{*}$. If none of these subwords represents an element of $\Omega_{1}^{*}$, then, by the Main Theorem, $X$ does not belong to [ $\left.\Omega\right]$. If however the subword $v_{i j}$ represents an element of $\Omega_{1}^{*}$, we determine a word $u_{i j}$ which represents the corresponding element of $\Omega^{*}$, and then determine the $\Pi$-length of the word

$$
w_{i j}=w_{\gamma(1)} \ldots w_{\gamma(j)} u_{i j}^{-1} w_{\gamma(j+1)} \ldots w_{\gamma(n)} \text {. }
$$

If for no such subword $v_{i j}$, the $\Pi$-length of $w_{i j}$ is strictly less than $n$, then, by the Main Theorem, $X$ does not belong to $[\Omega]$.

Now $w_{i j}$ represents an element of $[\Omega]$ if and only if $w^{\prime}$ represents an element of [ $\left.\Omega\right]$, so that if the $\Pi$-length of $w_{i j}$ is less than $n$, we have reduced the problem of determining whether or not $w$ represents an element of $[\Omega]$ to that of determining whether or not a word of smaller $\Pi$-length represents an element of $[\Omega]$. The process of repeating the steps described, each time with a word of smaller $\Pi$-length, must eventually terminate.

This proves the theorem.

\subsection{Examples illustrating the (infinite) word problem.}

Example 1. An infinite system for which the word problem is solvable.

An obvious example is the system with generators $x_{1}, x_{2}, x_{3}, \ldots$ and defining relations $x_{1}^{3}=1, x_{2}^{3}=1, x_{3}^{3}=1, \ldots$.

For the other examples we require the following result. 
LEMma 2.31. There is a one-one mapping $\phi$ of the set of positive integers into itself such that the image $\phi(n)$ of any given integer can be calculated, but there exists no finite process for deciding whether or not any given integer is an image under $\phi$.

Proof. $\dagger$ The proof depends on the result, proved by both Church and Turing, that there is no decision process for the restricted predicate calculus (cf. Kleene [3], p. 180 and p. 432). The formulae of the calculus are words in a set of symbols which can be assumed finite. Thus we can effectively enumerate the formulae $f_{1}, f_{2}, f_{3}, \ldots$ and also the finite sequences $S_{1}, S_{2}, S_{3}, \ldots$ of formulae. A sequence $S_{i}$ may or may not constitute a "proof" in the calculus. No decision process exists for determining whether or not any given formula $f_{i}$ can be "proved". Thus the function $\sigma$ given by

$$
\sigma(m)= \begin{cases}n & \text { if } S_{m} \text { is a proof of } f_{n}, \\ 0 & \text { if } S_{m} \text { is not a proof, }\end{cases}
$$

has the property that no finite process exists for determining whether or not any given positive integer is a value of $\sigma$.

Now define $\phi$ as follows. Let $\kappa(r)$ be the number of terms in the sequence $\sigma(1), \sigma(2)$, $\ldots, \sigma(r)$ which equal $\sigma(r)$ and put

$$
\phi(r)=2^{\sigma(r)} 3^{\kappa(r)} .
$$

Then $\phi$ is a one-one mapping of the set of positive integers into itself, and an integer of the form $2^{t}$. 3, where $t$ is a positive integer, is an image under $\phi$ if and only if $t$ is a value of $\sigma$. Thus $\phi$ has all the required properties.

Example 2. An infinite system for which the word problem is not solvable.

Consider the system with generators

$$
f_{n}, g_{n}, h_{n} \quad(n=1,2,3, \ldots)
$$

and defining relations

$$
f_{\phi(n)} h_{n}=1, g_{\phi(n)} h_{n}^{-1}=1 \quad(n=1,2,3, \ldots) .
$$

This system is explicitly given because every defining relation is of the form $f_{s} h_{r}=1$ or $g_{s} h_{r}^{-1}=1$ where $r$ and $s$ are positive integers, and if $r$ and $s$ are given, such a form is a defining relation if and only if $\phi(r)$, which can be calculated, equals $s$. The word problem is not solvable, for $f_{m} g_{m}=1$ follows from the defining relations if and only if $\ddagger$ there exists $n$ with $\phi(n)=m$, and we have seen that there is no finite process for deciding this.

In the remaining examples, the systems considered satisfy the conditions of the Main Theorem with (1.22) and the word problem is solvable for each constituent group. More precisely, the systems satisfy conditions (1), (2), (3) of $2 \cdot 2$, where in (3) they satisfy (1.22). Let us call such systems MT-systems.

Example 3. An MT-system in which

(i) every defining word has the same length,

(ii) no generator appears in more than two defining relations,

(iii) the word problem is not solvable.

† I am indebted to the referee for this proof. It is preferable to the author's proof (based on a recent result of Markov [4]) because it sets the lemma in its proper context.

$\$$ Here we use the result that if a generator $x$ does not appear in the defining relations, and if $w=1$ follows from the defining relations when $x$ appears in $w$, then the sum of the exponents $(+1$ or -1$)$ of $x$ in $w$ is zero. 
Let $p$ be an integer greater than 6 and take generators

$$
f_{s}(n), g_{s}(n) ; h(n) \quad(s=1,2, \ldots, p ; n=1,2, \ldots)
$$

with defining relations

$$
\left.\begin{array}{l}
f_{1}(\phi(n)) f_{2}(\phi(n)) \ldots f_{p}(\phi(n)) h(n)=1 \\
g_{1}(\phi(n)) g_{2}(\phi(n)) \ldots g_{p}(\phi(n)) h(n)^{-1}=1
\end{array}\right\}(n=1,2,3, \ldots),
$$

where $\phi(n)$ has the same meaning as in Lemma 2.31. Property (iii) is verified as in Example 2.

Example 4. An MT-system with the properties of Example 3 and in which, in addition, every generator appears in a defining relation.

In Example 3, add the generators

$$
x_{s}(t, n), y_{s}(t, n) \quad(s, t=1,2, \ldots, p ; n=1,2,3, \ldots)
$$

and add the defining relations

$$
\left.\begin{array}{l}
f_{s}(n) x_{s}(1, n) x_{s}(2, n) \ldots x_{s}(p, n)=1 \\
g_{s}(n) y_{s}(1, n) y_{s}(2, n) \ldots y_{s}(p, n)=1
\end{array}\right\}\left(\begin{array}{l}
s=1,2, \ldots, p \\
n=1,2,3, \ldots
\end{array}\right)
$$

Example 5. An MT-system in which

(i) every generator occurs in an infinite number of defining relations,

(ii) the II-lengths of the defining words are unbounded,

(iii) the numbers of cancellations between pairs of defining words are unbounded,

(iv) the word problem is solvable.

Consider the system with generators

$$
x_{n}, y_{n}, s_{n} \quad(n=1,2,3, \ldots),
$$

and defining relations $w_{n}=1(n=1,2,3, \ldots)$, where

$$
w_{n}=x_{1}^{2^{n}} y_{1}^{2^{n}} x_{2}^{2^{n}} y_{2}^{2^{n}} \ldots x_{3 n}^{2^{n}} y_{3 n^{2}}^{2^{n}} s_{1} s_{2} \ldots s_{n} .
$$

It is clear that $l\left(w_{n}\right)=7 n$, and that if $U_{n}$ and $U_{m}$ are cyclic arrangements corresponding to $w_{n}^{ \pm 1}$ and $w_{m}^{ \pm 1}$ respectively, then $\alpha\left(U_{n}, U_{m}\right) \leqslant \operatorname{Min}(n, m)$, so it is clear that the system is an MT-system, and (i), (ii), (iii) are satisfied. To prove (iv), it is sufficient to show that conditions (2B) and (2C) are satisfied (Theorem 2). Now a component of the form $x_{m}^{u}$ or $y_{m}^{u}(u \neq 0)$ occurs in a defining word or its inverse if and only if there exists a positive integer $n$ with the properties $u= \pm 2^{n}$ and $m \leqslant 3 n$. But any segment of length $n+1$ of the normal form of an element $U_{n}$ of $\Omega^{*}$ contains a component of this form ; hence so does a segment of length

$$
r\left(U_{n}\right)=7 n-3 n-1 \text {. }
$$

Thus (2B) and (2C) are satisfied.

Examples 3, 4 and 5 show that the conditions given by Stender ([2], p. 101) are neither necessary nor sufficient for the word problem to be solvable in an MT-system. The conditions may be put in the following form :

(1) the $\Pi$-lengths of the defining words are uniformly bounded,

(2) every generator occurs in only a finite number of defining words.

However, a sufficient set of conditions is obtained by adding a further condition, as in the following lemma.

Lemma 2.32. The word problem is solvable in an MT-system satisfying the conditions (1) and (2) and also 
(3) It can be decided whether or not a given generator $x$ appears in a defining relation, and if it does, then the (finite) set of defining relations in which it appears can be determined.

Proof. The elements of $\Omega_{1}^{*}$ are recognizable, because given any word $w$, we identify the finite number of defining relations containing the generators appearing in $w$ and it is then straightforward to determine whether or not $w$ represents an element of $\Omega_{1}^{*}$. If it does, we can easily determine the corresponding element of $\Omega^{*}$. On applying Theorem 2 , the lemma follows.

Examples 6 and 7. Example 6 will be an MT-system in which all the defining words have the same length $l$, where $l$ may be taken arbitrarily large, and the word problem is solvable. From this we shall obtain another system by altering at most one generator in each defining relation. The new system (Example 7) will also be an MT-system, but the word problem will not be solvable.

The interest of these examples is that they will show the necessity of condition (2B) (Theorem 2), which we may express by saying that the final segments of length $r(U)$ of the elements $U$ in $\Omega^{*}$ are recognizable.

Examples 6 and 7 are to be the first and second systems respectively in the following lemma.

LEMma 2.33. Let $\alpha, p$ be integers where $\alpha>1, p>6$, and let $l=p \alpha, r=l-3 \alpha-1$. Then two systems of generators and defining relations can be found with the following properties :

(i) Each system is an MT-system in which every element $X$ of $\Omega^{*}$ satisfies the conditions

$$
l(X)=l, \alpha(X)=\alpha
$$

and every element of $\Omega_{1}^{*}$, has length $r$ :

(ii) Each system satisfies (2C) (Theorem 2).

(iii) In the first system, the final segments of length $r$ of the elements in $\Omega^{*}$ are recognizable but the final segments of length $r-1$ are not recognizable. The word problem is solvable.

(iv) In the second system, the final segments of length $r+1$ of the elements in $\Omega^{*}$ are recognizable but the final segments of length $r$ are not recognizable. The word problem is not solvable.

Proof. The following system of generators and defining relations involves a function $\psi$, and the two systems required are obtained by taking

$$
\psi(n)=\left\{\begin{array}{ll}
n & \text { for the first system, } \\
\phi(n) & \text { for the second system. }
\end{array} \quad(n=1,2,3, \ldots)\right.
$$

The system has generators

$$
\left.\begin{array}{c}
a_{1}(s, n), a_{2}(s, n), \ldots, a_{\alpha-1}(s, n), \\
b_{1}(s, n), b_{2}(s, n), \ldots, b_{\alpha-1}(s, n), \\
x_{s}(n), z_{s}(n), \\
y_{s}, \\
h_{2}(s, n), h_{3}(s, n), \ldots, h_{r}(s, n),
\end{array}\right\}\left(\begin{array}{l}
s=1,2, \ldots, p ; \\
n=1,2,3, \ldots
\end{array}\right)
$$

and, in the notation

$$
\left.\begin{array}{l}
A_{s}(n)=a_{1}(s, n) \ldots a_{\alpha-1}(s, n) \\
B_{s}(n)=b_{1}(s, n) \ldots b_{\alpha-1}(s, n) \\
H_{s}(n)=h_{2}(s, n) \ldots \ldots h_{r}(s, n)
\end{array}\right\}
$$


it has defining relations

$$
\left.\begin{array}{c}
A_{1}(n) x_{1}(n) \ldots A_{p}(n) x_{p}(n)=1 \\
B_{t}(n)^{-1} x_{t-1}(n)^{-2} A_{t}(n) x_{t}(n)^{3} B_{t+1}(n) y_{p-t}^{c(n)} z_{p-t+1}(\psi(n)) H_{p-t+1}(\phi(n)) y_{p-t+1}^{d(n)}=1 \\
(t=1,2, \ldots, p ; n=1,2,3, \ldots)
\end{array}\right\}
$$

where

$$
c(n)=2^{n}, d(n)=3-2^{n}
$$

and

$$
x_{0}(n)=x_{p}(n), \quad y_{0}=y_{p}, \quad B_{p+1}(n)=B_{1}(n) .
$$

We prove first that both systems are MT-systems. By the definitions of $l$ and $r$, it follows that every element of $\Omega^{*}$ has length $l$. To show that $\alpha(U, V) \leqslant \alpha$ we proceed as follows. If $U, V$ have different arguments $n, n^{\prime}$, then no cancellations can occur in the product $U$. $V$, but there might be an amalgamation between the generators $y_{s}$, so $\alpha(U, V) \leqslant 1$. If $U, V$ both have argument $n$, then among the generators $a_{i}(s, n)$ (or $b_{i}(s, n)$ ) there can occur up to $\alpha-1$ cancellations, followed possibly by an amalgamation between generators $x_{s}(n)$ (or $y_{s}$ ), but among the generators $x_{s}(n), z_{s}(\psi(n)), y_{s}, h_{i}(s, \phi(n))$ no cancellations can occur. It is now clear also that $\alpha(X)=\alpha$ for $X \epsilon \Omega^{*}$. The constituent groups are all infinite cyclic groups; hence the word problem is solvable for each of them.

We now prove (ii), (iii), (iv). The set of words $H_{p-t+1}(\phi(n))(t=1,2, \ldots, p ; n=1,2$, $3, \ldots)$ is not recognizable, since a word of the form $H_{p-t+1}(m)$ lies in the set if and only if $m$ is the image of an integer under $\phi$. On the other hand the set of words $z_{p-t+1}(n) H_{p-t+1}(\phi(n))$ $(t=1,2, \ldots, p ; n=1,2,3, \ldots)$ is recognizable because a word of the form $z_{p-t+1}(n) H_{p-t+1}(m)$ lies in the set if and only if $\phi(n)$, which can be calculated, equals $m$. It will now be seen that for the first system, the final segments of length $r$ of the elements in $\Omega^{*}$ are recognizable but the final segments of length $r-1$ are not recognizable. But the first system satisfies (2C), so Theorem 2 is applicable and shows that the word problem is solvable.

To establish the lemma, it is only necessary to prove that the second system satisfies (2C) and the word problem is not solvable.

Now any element of $\Omega_{1}^{*}$ has length $r$, so a given element $X$ of $\Omega_{1}^{*}$ either contains a generator of the form $a_{i}(s, n), b_{i}(s, n)$ or $x_{s}(n)$ (or the inverse of such a generator), or a component of the form $y_{a}^{u}$, where $\pm u=2^{n}$ or $3-2^{n}$, or else it has the form

$$
\left(z_{p-t+1}(m) H_{p-t+1}(m)\right)^{\kappa} \quad(\kappa= \pm 1) .
$$

In the first case the integer $n$ leads us to the element of $\Omega^{*}$ corresponding to $X$. In the second case we must evaluate successively $\phi(i)(i=1,2,3, \ldots)$ until the integer $n$ is reached for which $\phi(n)=m$. This process will terminate because $X$ is given to be an element of $\Omega_{1}^{*}$, so such an $n$ exists. Having found $n$, we can easily determine the required element of $\Omega^{*}$.

By considering only the defining relations with a fixed value of $n$, we see that the relation

$$
\prod_{t=1}^{p}\left(y_{p-t}^{c(n)} z_{p-t+1}(\psi(n)) H_{p-t+1}(\phi(n)) y_{p-t+1}^{d(n)}\right)^{-1}=1
$$

and hence the relation

$$
\prod_{s=1}^{p} z_{s}(\psi(n)) H_{s}(\phi(n)) y_{s}^{3}=1
$$

follow from the defining relations. But $\psi(n)=\phi(n)$, so we can use an argument similar to that in Example 2 to show that it is impossible to decide whether or not relations of the form 


$$
\prod_{s=1}^{p} z_{s}(m) H_{s}(m) y_{s}^{3}=1
$$

follow from the defining relations. Thus the word problem is not solvable for the second system and the lemma is proved.

\section{APPENDIx [added 21st March 1956]}

Condition $(2 C)$ can be omitted from Theorem 2.

The meaning of condition $(2 \mathrm{C})$ was given in $2 \cdot 2$. Using the same notation, we have to find a finite process $Q$ which, applied to any word $w$ representing an element $U_{1}$ of $\Omega_{1}^{*}$, determines a word representing the (unique) element $U$ of $\Omega^{*}$ corresponding to $U_{1}$. Let $\mathcal{P}$ be the finite process which ensures that $R^{\prime}$ is explicitly given. By the meaning of a finite process $(\$ 2 \cdot 1$, footnote) it may be assumed that the domain of $P$, that is, the set of objects which can be tested by $\mathcal{P}$, is a countable set which can be enumerated explicitly as a sequence, say $D_{1}$, $D_{2}, \ldots$ The required process $\mathbb{Q}$ can now be described. We apply $\mathcal{P}$ to $D_{1}, D_{2}, \ldots$ successively and every time it is found that $D_{i}$ is an element of $R^{\prime}$ we determine whether or not $D_{i}$ corresponds to $w$. Thus writing $D_{i}$ in the form $w_{i}=1$ we consider all the cyclic arrangements of $w_{i}$ and $w_{i}^{-1}$ and determine whether or not any one of them represents the element $U$. Now every element of $R^{\prime}$ occurs in the sequence $D_{1}, D_{2}, \ldots$ so the process does eventually terminate.

\section{REFERENCES}

1. Church A., A note on the Entscheidungsproblem, $J$. Symbolic Logic, 1 (1936), 40-41. Correction, ibid, 101-102.

2. Church, A., An unsolvable problem of elementary number theory, Amer. J. Math., 58 (1936), 345-363.

3. Kleene, S. C., Introduction to Metamathematics (Amsterdam, 1952).

4. Markov, A., On an unsolvable problem concerning matrices, Doklady Akad. Nauk, SSSR, N.S. 78 (1951), 1089-1092. $103-105$.

5. Post, E. L., Finite combinatory processes-formulation 1, J. Symbolic Logic, 1 (1936),

6. Stender, P. V., On the application of the sieve method to the solution of the word problem for certain groups with a denumerable set of generating elements and a denumerable set of defining relations, Mat. Sbornik, N.S. 32 (74) (1953), 97-108.

7. Tartakovskii, V. A., The sieve method in group theory, Mat. Sbornik, N.S. 25 (67) (1949), 3-50.

8. Tartakovskil, V.A., Application of the sieve method to the solution of the word problem for certain types of groups, Mat. Sbornik, N.S. 25 (67) (1949), 251-274.

9. Tartakovskil, V. A., Solution of the word problem for groups with a $k$ reduced basis for $k>6$, Isvestiya Akad. Nauk, SSSR, Ser. Mat., 13 (1949), 483-494.

10. Amer. Math. Soc. Translation No. 60 (1952), (Translation of Tartakovskir [7], [8] and [9]).

11. Turing, A. M., On computable numbers, with an application to the Entscheidungsproblem, Proc. London Math. Soc. (2), 42 (1937), 230-265. Correction, ibid, 43 (1937), 544-546.

12. Schiek, H., Ähnlichkeitsanalyse von Gruppenrelationen-to appear in Acta Mathematica. .

\section{The University}

Glasgow 